\title{
Analysis of 30 Spinal Angiograms Falsely Reported as Normal in 18 Patients with Subsequently Documented Spinal Vascular Malformations
}

\author{
(D) P. Barreras, DD. Heck, (DB. Greenberg, (D).-P. Wolinsky, (D) C.A. Pardo, and (DP. Gailloud
}

\begin{abstract}
BACKGROUND AND PURPOSE: The early diagnosis of spinal vascular malformations suffers from the nonspecificity of their clinical and radiologic presentations. Spinal angiography requires a methodical approach to offer a high diagnostic yield. The prospect of falsenegative studies is particularly distressing when addressing conditions with a narrow therapeutic window. The purpose of this study was to identify factors leading to missed findings or inadequate studies in patients with spinal vascular malformations.
\end{abstract}

MATERIALS AND METHODS: The clinical records, laboratory findings, and imaging features of 18 patients with spinal arteriovenous fistulas and at least 1 prior angiogram read as normal were reviewed. The clinical status was evaluated before and after treatment by using the Aminoff-Logue Disability Scale.

RESULTS: Eighteen patients with 19 lesions underwent a total of 30 negative spinal angiograms. The lesions included 9 epidural arteriovenous fistulas, 8 dural arteriovenous fistulas, and 2 perimedullary arteriovenous fistulas. Seventeen patients underwent endovascular (11) or surgical (6) treatment, with a delay ranging between 1 week and 32 months; the Aminoff-Logue score improved in 13 (76.5\%). The following factors were identified as the causes of the inadequate results: 1) lesion angiographically documented but not identified (55.6\%); 2) region of interest not documented (29.6\%); or 3) level investigated but injection technically inadequate (14.8\%).

CONCLUSIONS: All the angiograms falsely reported as normal were caused by correctible, operator-dependent factors. The nonrecognition of documented lesions was the most common cause of error. The potential for false-negative studies should be reduced by the adoption of rigorous technical and training standards and by second opinion reviews.

ABBREVIATIONS: PmAVF = perimedullary arteriovenous fistula; SDAVF $=$ spinal dural arteriovenous fistula; SEAVF $=$ spinal epidural arteriovenous fistula

S pinal vascular malformations can be subdivided into highflow and low-flow lesions. The high-flow group includes spinal arteriovenous malformations and prominent perimedullary arteriovenous fistulas (PmAVF, Merland type 2 and $3^{1}$ ). The lowflow group is principally made of spinal dural (SDAVF) and spinal epidural arteriovenous fistulas (SEAVF) as well as less common small PmAVFs (Merland type 1). Although the detection of fastflow lesions is generally straightforward with noninvasive imaging because of the large size of the vessels involved, low-flow anomalies_-which are more frequent_can represent a major

Received December 30, 2016; accepted after revision April 24, 2017.

From the Transverse Myelitis Center, Department of Neurology (P.B., C.A.P.), Division of Interventional Neuroradiology (D.H., P.G.), and Department of Neurosurgery (I.P.W.), The Johns Hopkins Hospital, Baltimore, Maryland; and Transverse Myelitis Center (B.G.), University of Texas Southwestern Medical Center, Dallas, Texas.

Please address correspondence to Philippe Gailloud, MD, Johns Hopkins University School of Medicine, 1800 E Orleans St, Bloomberg Building Room 7216, Baltimore, MD 21287; e-mail: phg@jhmi.edu

http://dx.doi.org/10.3174/ajnr.A5275 diagnostic challenge. Low-flow arteriovenous fistulas typically occur in older men presenting with a venous hypertensive myelopathy secondary to the drainage of the fistula into the perimedullary venous system. ${ }^{2-5}$ They are characterized by progressive courses and narrow therapeutic windows, yet their diagnoses are often established with considerable delay, if at all. ${ }^{5}$ This situation is in large part related to their nonspecific clinical presentations and the ambiguous noninvasive imaging characteristics of venous hypertensive myelopathy. Spinal DSA, the "gold standard" technique for the investigation of spinal vascular anomalies, remains necessary to identify and characterize these lesions. Modern spinal DSA is associated with extremely low risks of neurologic or systemic complications, ${ }^{6}$ but it is a technically demanding procedure that requires a methodical approach to offer a high diagnostic yield.

This article analyzes the clinical and imaging characteristics of 18 patients with proved spinal vascular malformations but initial angiographic investigation reported as normal. The purpose of this review was to identify the factors that led to missed findings or 
inadequate studies and propose strategies aimed at reducing their impact.

\section{MATERIALS AND METHODS}

This is a retrospective study including 18 patients treated at our institution (The Johns Hopkins Hospital) between August 2009 and April 2016 for a vascular malformation and having at least 1 initial spinal angiogram read as normal. The final angiographic study unequivocally documenting the vascular malformation was performed by the senior author (P.G.) in all cases; the procedures were all performed under conscious sedation. The negative studies were reviewed and classified by 2 authors who were both aware of the final angiographic diagnoses (P.B., P.G.). In situations combining multiple sources of error, the visibility of the lesion was used as the dominant criterion to categorize the cause of the erroneous result. In case 15 for example, the quality of the angiographic image was reduced by the nonselective nature of the injection, yet the lesion was documented; this case was classified as a documented but not identified lesion.

Seventeen patients had their vascular malformations treated, including 1 patient with 2 separate anomalies, whereas 1 lesion

\section{Table 1: Aminoff-Logue Disability Scale}

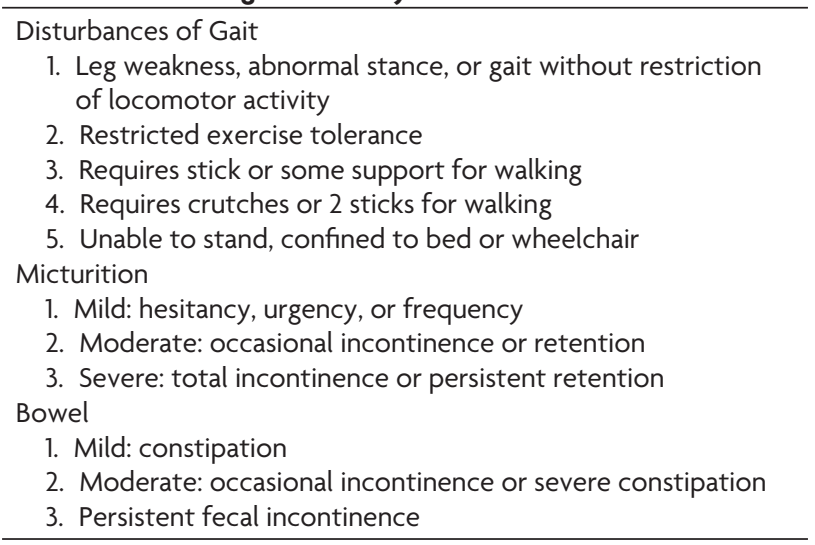

spontaneously resolved without intervention. The clinical status of each patient was evaluated before treatment and at the last follow-up visit by using the Aminoff-Logue Disability Scale based on data gathered retrospectively from chart review (Table 1). ${ }^{7}$ Updated follow-up information was obtained by telephone interviews. The difference between the mean preand posttreatment Aminoff-Logue scores was calculated by using a standard Student $t$ test. A $P$ value $<.05$ was used to define statistical significance.

Besides the presence of an abnormal medullary signal on T2weighted images, 2 additional MR imaging characteristics were recorded: the presence of parenchymal enhancement after gadolinium administration and flow voids on T2-weighted images, categorized as absent $(-)$, subtle $(+)$, focal $(++)$, or diffuse $(+++)($ Fig 1$)$.

\section{RESULTS}

\section{Demographic and Clinical Features}

We found 18 patients with 19 angiographically confirmed spinal vascular malformations and at least 1 angiographic study read as normal, for a total of 30 negative angiograms (median, 1; average, 1.7; range, 1-6) (Table 2). The cohort included 15 men and 3 women, aged from 25-89 years. Most patients were men (83\%) with an average age of 63 years at the time of presentation. Past medical history - available for all patients—included cardiovascular risk factors, notably arterial hypertension in 12 cases (67\%) and smoking in 11 cases $(61 \%)$.

The following symptoms and clinical features were recorded in the patients' charts: leg weakness $(n=17 ; 94 \%)$, leg numbness ( $n=12 ; 67 \%)$, bladder dysfunction $(n=12 ; 67 \%)$, neurogenic claudication $(n=7 ; 39 \%)$, abnormal sensory examination ( $n=$ $16 ; 89 \%)$, and a sensory level noted in 11 cases (61\%). Urinary retention and/or decreased rectal tone was noted in 7 cases (39\%). The beginning of clinical manifestation according to the chart was greater than 21 days in 15 patients (83\%), less than 48 hours in 1 (5\%), and acute in $2(11 \%)$.

The CSF - collected in 14 patients -

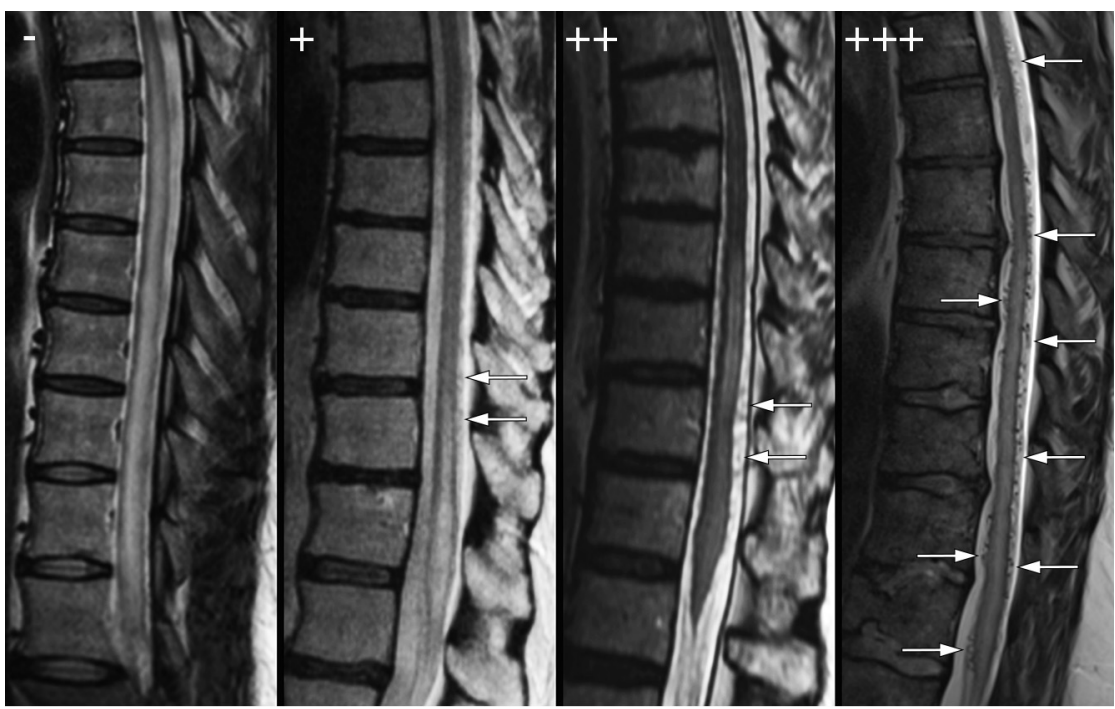

FIG 1. Grading of flow voids on sagittal T2-weighted images $(-,+,++,+++)$. Left, 50 -year-old man (patient 2) with a left L5 SDAVF. Center left, 73-year-old man (patient 8) with a left L4 SEAVF. Center right, 75-year-old man (patient 10) with a right T12 SDAVF. Right, 49-year-old man (patient 12) with a right S2 SDAVF. showed elevated protein $(>45 \mathrm{mg} / \mathrm{dL})$ in 11 patients $(79 \%)$ and pleocytosis $\left(>5\right.$ cells $\left./ \mathrm{mm}^{3}\right)$ in $5(36 \%)$.

The initial diagnosis was transverse myelitis in 15 cases (83\%). Of the 13 patients initially treated with IV methylprednisolone, 1 improved (8\%), 7 showed no response (54\%), and 5 experienced immediate and substantial clinical worsening $(38 \%)$.

\section{Noninvasive Imaging \\ Characteristics}

MR imaging documented a myelopathy extending over at least 4 vertebral levels, with a central pattern of abnormal signal involving the thoracic and/or lumbar spinal cord in all patients. Enhancement after the gadolinium injection was noted in 15 cases $(83 \%)$. Flow voids were present in 11 cases $(61 \%)$. They were focal 


\begin{tabular}{|c|c|c|c|c|c|c|c|c|c|c|}
\hline \multirow[b]{2}{*}{ Case } & \multirow[b]{2}{*}{ Age/Sex } & \multirow[b]{2}{*}{ Gad } & \multirow[b]{2}{*}{ Flow Voids } & \multirow[b]{2}{*}{ Initial Diagnosis } & \multirow[b]{2}{*}{ Negative DSA } & \multirow[b]{2}{*}{ Final Diagnosis } & \multirow[b]{2}{*}{$\operatorname{Loc}^{a}$} & \multirow[b]{2}{*}{ Treat } & \multicolumn{2}{|c|}{$\begin{array}{c}\text { Aminoff-Logue } \\
\text { Score }\end{array}$} \\
\hline & & & & & & & & & Pre & Post \\
\hline 1 & $40 / \mathrm{M}$ & + & - & $\mathrm{TM}$ & 2 & SEAVF & T9 & Surg & 8 & 6 \\
\hline 2 & $50 / \mathrm{M}$ & + & - & TM & 2 & SDAVF & L5 & Endo & 8 & 8 \\
\hline 3 & $73 / \mathrm{M}$ & + & + & TM & 1 & SDAVF & L4 & Endo & 8 & 5 \\
\hline 4 & $80 / \mathrm{M}$ & + & ++ & TM & 2 & SEAVF & L5 & Endo & 9 & 7 \\
\hline 5 & $60 / \mathrm{M}$ & & + & TM & 1 & SDAVF & $\mathrm{T} 4$ & Endo & 9 & 8 \\
\hline \multirow[t]{2}{*}{$6^{b}$} & $56 / \mathrm{F}$ & + & + & TM & 1 & SEAVFs & L2 & Endo & 10 & 8 \\
\hline & & & & & & & L4 & Endo & & \\
\hline 7 & $89 / \mathrm{M}$ & & - & Tumor & 1 & SEAVF & S1 & Endo & 3 & 1 \\
\hline 8 & $73 / F$ & + & - & $\mathrm{TM}$ & 1 & SEAVF & L4 & Endo & 10 & 5 \\
\hline 9 & $58 / \mathrm{M}$ & + & + & TM & 1 & PmAVF & $\mathrm{T} 10$ & Surg & 10 & 10 \\
\hline 10 & $75 / \mathrm{M}$ & + & ++ & TM & 1 & SDAVF & $\mathrm{T} 12$ & Endo & 10 & 7 \\
\hline 11 & $75 / \mathrm{M}$ & + & - & TM & 3 & SDAVF & L3 & Surg & 8 & 9 \\
\hline 12 & $49 / \mathrm{M}$ & + & ++ & TM & 6 & SDAVF & S2 & Endo & 6 & 4 \\
\hline 13 & $69 / \mathrm{M}$ & & - & $\mathrm{TM}$ & 2 & SEAVF & L5 & Endo & 11 & 11 \\
\hline 14 & $56 / \mathrm{M}$ & + & ++ & Syrinx & 1 & PmAVF & $\mathrm{T} 8$ & Surg & 5 & 1 \\
\hline 15 & $66 / F$ & + & - & $\mathrm{TM}$ & 1 & SDAVF & $\mathrm{T} 10$ & Endo & 11 & 7 \\
\hline 16 & $71 / M$ & & + & $\mathrm{TM}$ & 2 & SEAVF & $\mathrm{S} 1$ & None & 10 & None \\
\hline 17 & $61 / \mathrm{M}$ & + & - & NMO & 1 & SDAVF & $\mathrm{T} 4$ & Surg & 9 & 6 \\
\hline 18 & $25 / \mathrm{M}$ & + & ++ & $\mathrm{TM}$ & 1 & SEAVF & $\mathrm{T} 12$ & Surg & 11 & 4 \\
\hline
\end{tabular}

Note:-Endo indicates endovascular; Gad, spinal cord enhancement after gadolinium; Loc, lesion location; NMO, neuromyelitis optica; Pre, pretreatment; Post, posttreatment; Surg, surgery; TM, transverse myelitis; Treat, treatment.

a For the 2 PmAVFs, the indicated level corresponds to the feeding artery.

${ }^{b}$ Patient 6 had 2 separate SEAVFs treated in separate endovascular procedures.

$(++)$ in 4 cases $(22 \%)$ and diffuse $(+++)$ in $2(11 \%)$. Subtle flow voids $(+)$ were retrospectively observed in 5 instances (28\%), but reported only once.

\section{Vascular Malformation Characteristics}

All the lesions were low-flow arteriovenous fistulas, including 9 SEAVFs (47\%), 8 SDAVFs (42\%), and 2 PmAVFs (Merland Type $1 ; 1 \%$ ) (Table 2). No spinal arteriovenous malformation or prominent PmAVF (Merland Type 2 or 3) was observed. The delay between presentation and treatment ranged between 1 week and 32 months (median, 9.0; average, 11.1).

Both PmAVFs were located on the anterior aspect of the tip of the conus medullaris and were supplied by the anterior spinal artery via anterior radiculomedullary arteries originating from a thoracic intersegmental artery (T8 and T10, respectively). The SDAVF/SEAVFs were thoracic (T2 through T12) $(n=6 ; 35.3 \%)$, lumbar (L1 through L5) $(n=8 ; 47.1 \%)$, or sacral (S1 through S2) $(n=3 ; 17.6 \%)$. Nine of these 17 lesions $(52.9 \%)$ were located at L4 or below, including 6 supplied either by an iliolumbar artery $(n=3)$ or by a lateral sacral artery branch $(n=3)$.

Eighteen of the 19 lesions were treated either endovascularly $(n=12 ; 66.7 \%)$ or surgically $(n=6 ; 33.3 \%)$. One patient had 2 separate SEAVFs; another patient had a pelvic SEAVF that thrombosed spontaneously.

After treatment, the clinical condition measured by the Aminoff-Logue scale improved in 13 of the 17 patients $(76.5 \%)$, with an average gain of 3 points $(P=.01)$ (Table 2$)$. Three patients were unchanged $(17.6 \%)$, and 1 got worse immediately after surgery, likely as a result of spinal venous thrombosis. Of note, 5 patients reported a subjective improvement in their pain level as well as increased sensation in previously numb areas, 2 elements not captured by the AminoffLogue scale. The patient with the spontaneously thrombosed lesion showed no clinical improvement.

\section{Analysis of Missed Findings or Inadequate Studies}

Thirty spinal angiograms falsely reported as normal were obtained in 18 patients (median, 1; average, 1.7; range, 1-6), 26 of them performed in various academic centers (86.7\%). Twentyseven of these angiograms were available for review. The factors identified as the cause of the missed findings or inadequate studies could be categorized as follows:

1) The lesion was angiographically documented but not identified ( $n=15 ; 55.6 \%)$. In some instances, the anomaly went unnoticed (Fig 2), whereas in others, the abnormal vessel was seen but misinterpreted (Fig 3).

2) The region of interest was not documented ( $n=8 ; 29.6 \%)$, including: a) the level of interest was not investigated $(n=7$; $25.9 \%$ ), the lesion being located at or below L3 in all instances ( $\mathrm{L} 3=1, \mathrm{~L} 4=2, \mathrm{~L} 5=3, \mathrm{~S} 2=1)$; or b) the level was accidentally skipped $(n=1 ; 3.7 \%)$.

3) The level was investigated, but the injection was technically inadequate $(n=4 ; 14.8 \%)$. In 3 cases, the supplying branch was not studied selectively (Fig 4), whereas in 1 of the 2 PmAVFs, the anterior spinal artery was well opacified, but the lesion was not included in the field-of-view.

As noted earlier, in situations combining multiple technical and/or cognitive factors, the lesion's visibility was used as the dominant criterion to categorize the cause of the false-negative study.

\section{DISCUSSION}

\section{Patient Demographics and Type of Vascular Malformations}

This report analyzed 30 spinal angiograms read as normal in 18 patients later diagnosed with a spinal vascular malformation. All of the lesions diagnosed were slow-flow vascular malformations; besides 2 small PmAVFs (Merland Type 1; 10\%) located at the conus medullaris, they were either SEAVFs or SDAVFs. This finding is not surprising because the detection of conspicuous anom- 

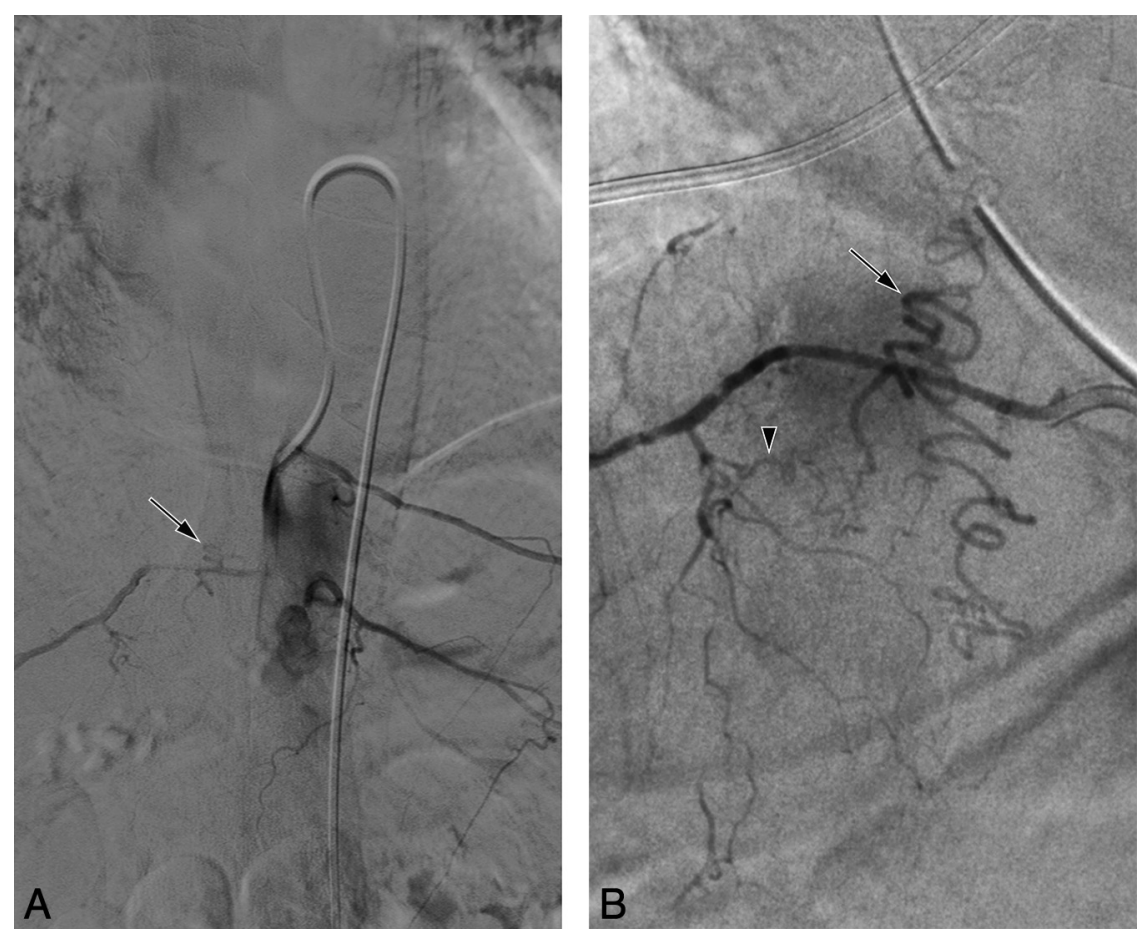

FIG 2. 66-year-old woman (patient 15) with a right T10 SDAVF and 1 prior negative spinal angiogram. A, DSA, nonselective injection at the T9 level, posteroanterior view, arterial phase (first study). The right $\mathrm{T} 10$ intersegmental artery supplies an arteriovenous shunt with early opacification of perimedullary venous structures (arrow). The anomaly was not detected. B, DSA, right T10 injection, posteroanterior view, arterial phase (second study). Selective angiography confirms the presence of an SDAVF fed by the right T10 radicular artery (arrowhead) and documents its extensive perimedullary drainage (arrow).
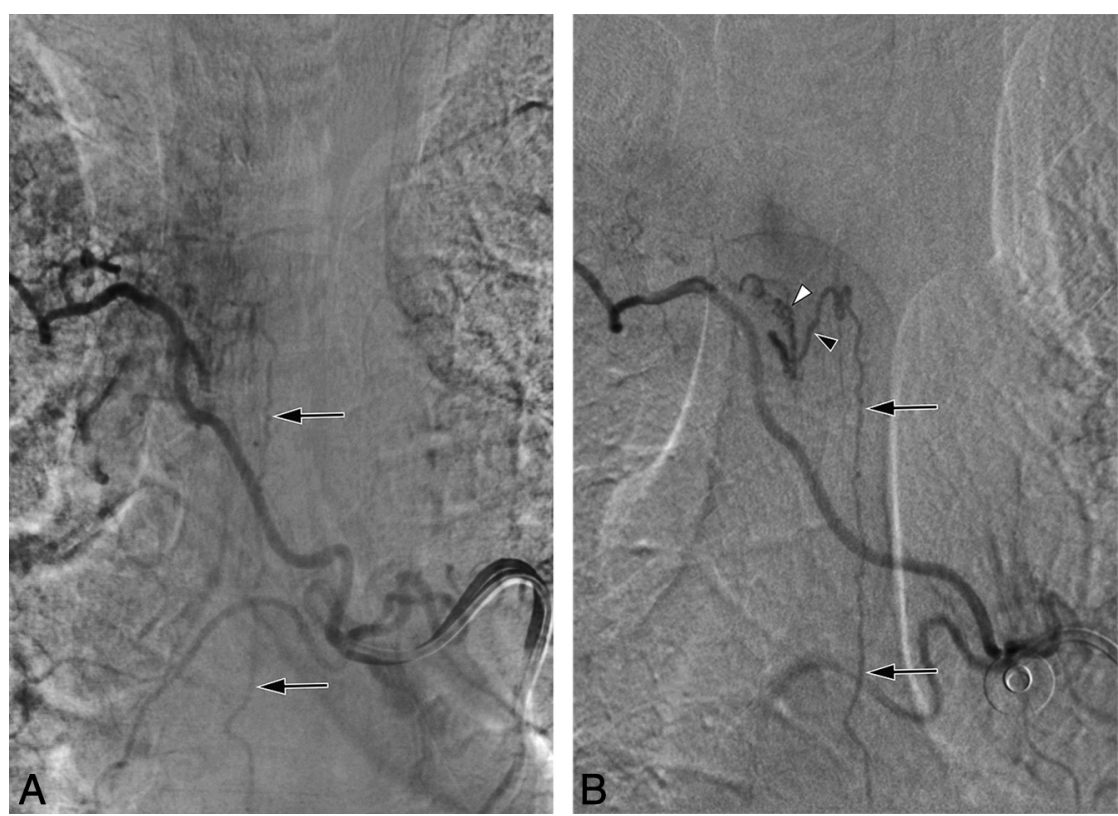

FIG 3. 60-year-old man (patient 5) with a right T4 SDAVF and 1 prior negative angiogram. The patient consulted for a second opinion after substantial clinical pejoration during intravenous steroid therapy. A, DSA, right T4 injection, posteroanterior view, arterial phase (first study). A midline vessel (arrows) was noted but interpreted as being the anterior spinal artery. B, DSA, right T4 injection, posteroanterior view, arterial phase (second study). A right T4 radiculomeningeal branch (white arrowhead) supplies an arteriovenous shunt draining into a posterior radiculomedullary vein (black arrowhead) and the posterior-median spinal vein (black arrows). alies such as spinal arteriovenous malformations or PmAVFs (Merland Type 2 or 3 ) does not usually represent a challenge for noninvasive imaging, spinal DSA being then used to characterize their angioarchitecture and plan their management.

Our patients' demographic characteristics (predominantly older men) are consistent with previously published data on slow-flow vascular malformations. ${ }^{5}$ From a diagnostic perspective, the high prevalence of elevated CSF proteins $(79 \%)$ and gadolinium enhancement on MR imaging (83\%) probably explains the frequent misdiagnosis of transverse myelitis $(83 \%) .{ }^{8}$ Flow voids, often considered as essential to the diagnosis of vascular malformation, were retrospectively detected in 11 cases (61\%) but, because of their subtle nature, were correctly identified at the time of initial MR imaging interpretation in only 7 cases $(39 \%)$.

Of particular interest is the preponderance of low lumbar and sacral lesions in our cohort; most of the missed SEAVFs/SADVFs (52.9\%) were found at or below the L4 level. Besides certain technical considerations discussed separately, this finding seems to correlate with the relatively common misconception that a thoracic myelopathy cannot be accounted for by a pelvic lesion.

In terms of functional outcome, it is important to note that despite the significantly delayed diagnosis (11 months on average) and the clinical worsening caused in some cases by inadequate initial measures (notably steroid therapy), patients did still significantly benefit from the treatment of their vascular malformation. First and foremost, the progressive clinical deterioration typically associated with venous hypertensive myelopathy was stopped in all cases but 1 , the exception being a patient who got worse shortly after treatment, likely from spinal venous thrombosis. Besides stopping the disease progression, endovascular or surgical therapy was able to bring a functional improvement in 13 of the 17 patients treated, with a significant difference retrospectively noted between the pre- and posttreatment Aminoff-Logue scores retrospectively (mean follow-up, 20 months; $P=.012$ ). This finding is consistent with the previously 

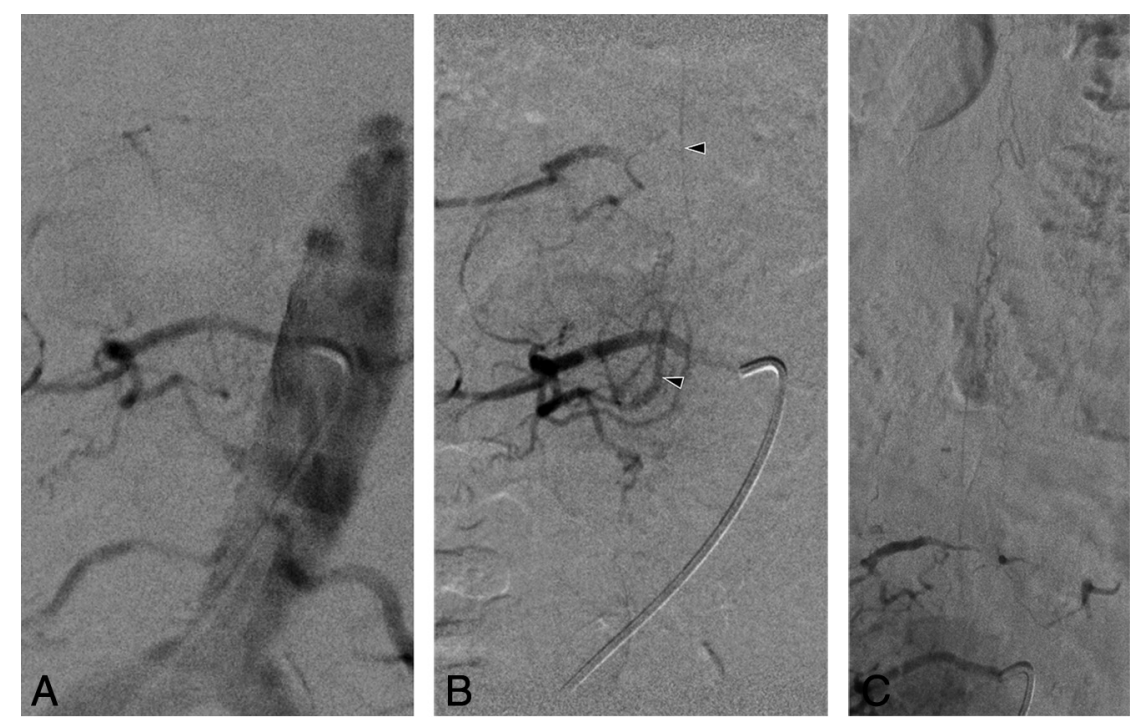

FIG 4. 75-year-old man (patient 11) with a right L3 spinal dural arteriovenous fistula (SDAVF) and 3 prior negative angiograms. A, DSA, nonselective injection at the level of $L 3$, posteroanterior view, arterial phase (first study), showing no evidence of vascular malformation. In 1 of the other negative angiograms, L3 was not investigated at all, and the third study was not available for review. B, DSA, right L3 injection, posteroanterior view, arterial phase (second study), an arteriovenous shunt supplied by a right $\mathrm{L} 3$ radiculomeningeal branch and draining into a right $\mathrm{L} 3$ radiculomedullary vein (arrowheads). C, DSA, right L3 injection, posteroanterior view, arterial phase (second study). The congested perimedullary venous system surrounding the lumbosacral spinal cord is documented by this acquisition with a larger field of view.

held view that even patients with severe disabilities can show significant improvement. ${ }^{9}$

\section{Technical Factors Leading to Missed Findings or Inadequate Studies}

This review identified several factors behind the missed findings or inadequate results. All were operator-dependent and potentially avoidable. The principal cause of missed diagnoses was a documented yet unrecognized lesion (55.6\%). In other words, the anomaly was visible in the recorded images, but not identified as a vascular malformation. The same mechanism was responsible for false-negative studies in 3 additional cases of SDAVFs not included in our cohort because of a lack of detailed clinical information. This finding serves as a strong reminder of the complexity of spinal angiographic images, considering notably that most studies $(86.7 \%)$ were obtained in academic centers.

The vessel of interest not being investigated was the second most frequent cause of inadequate study (29.6\%). In 1 case, the vertebral levels were mislabeled and the feeding artery skipped by mistake. In the other 7 instances (25.9\%), the intersegmental artery supplying the lesion was not included in the planned angiogram. Interestingly, the lesion was located at or below L3 in all of these cases. This finding has an important implication besides the fact that low lumbar or sacral vascular malformations are frequent; it suggests that low lumbar and pelvic vessels are often ignored during spinal angiography. Although this may be partly attributed to the technically more demanding catheterization of these branches, notably in older patients, the main reason behind this disregard appears to be the misconception that a pelvic flush angiogram supplemented by selective internal iliac injections is not an integral component of a full spinal angiogram. In our practice, spinal DSA performed in older patients with a suspicion of low-flow vascular malformation starts with a pelvic flush angiogram, which not infrequently establishes the diagnosis during the first few minutes of the procedure. Finally, in 4 cases $(14.8 \%)$, the vessel of interest was investigated, but the angiograms were technically inadequate. In 1 of the PmAVFs, the feeding artery (left T10) was injected, but considered as normal, and the conus medullaris (site of the lesion) was not included in the field of view. In the other 3 instances, the feeding vessels were studied with nonselective injections, the small amount of contrast passing through the vessel of interest being considered of diagnostic value. In each case, a lesion was subsequently identified by selective angiography.

\section{How to Limit the Risk of Inadequate Spinal Angiograms?}

Our findings suggest that missed findings or inadequate studies might be avoided in most cases by the adoption of a rigorous angiographic protocol. The following recommendations-easily implementable in daily angiographic practices - should significantly reduce the risk of falsenegative studies:

1) Carefully identify the vertebral levels investigated at the beginning of the procedure. Although the use of a ruler is in our opinion mandatory, it may not prevent skipping a level if one does not meticulously document the progress of the study and record the explored branches.

2) Avoid, as much as possible, poor angiographic visualization related to nonselective injections. ${ }^{10}$ It is also critical to document radiculomedullary arteries in their entirety, as exemplified by the PmAVF left out of the field of view during the injection of the artery of Adamkiewicz in 1 of the negative angiograms.

3) In most instances, it is necessary to perform a complete spinal angiogram - even if a lesion has already been documented - including the pelvis and the cervical region and, in selected cases, the carotid arteries as well. There is no strict correlation between the site of an arteriovenous fistula and the location and extent of the resulting spinal cord damage. A cervical SDAVF can, for example, induce midthoracic or lumbar cord edema with a normal MR imaging appearance of the cervical and upper thoracic spinal parenchyma. The potential for multiple synchronous spinal lesions must also be kept in mind.

4) Finally, the fact that most of the missed lesions were actually documented by the initial "negative" studies emphasizes the importance of a meticulous analysis of angiographic images, optimized by electronic means such as best mask selection and pixel shifting. It also stresses the value of second readings and second opinions. Second opinions also represent an educative tool, in keeping with Pinto and Brunese's ${ }^{11}$ opinion that "a radiology safety culture will only exist when the radiologist who made the 
error views such feedback positively as a learning experience.” With angiographic images as complex and findings as subtle as the ones acquired during spinal angiography, no level of proficiency can prove completely immune to faulty interpretation.

\section{Spinal Angiography as the "Gold Standard" Spinal Vascular Technique}

Although no imaging technique is exempt from the risk of falsenegative studies, it seems that various technical and cognitive factors render spinal angiography more prone to misleading interpretation than, for example, cerebral angiography. This fact directly impacts patient care because there is a justified tendency to rely on a "gold standard" technique. The number of patients with vascular malformations who remain misdiagnosed after a spinal angiogram falsely read as normal is unknown but likely not anecdotal. Spinal angiography should therefore only be considered as a "gold standard" technique when performed by experienced angiographers following strict technical guidelines.

\section{CONCLUSIONS}

In the spinal angiograms analyzed for this study, the causes of missed findings or inadequate studies were all avoidable operatorrelated factors, most notably the nonrecognition of an angiographically documented lesion. The potential for inadequate studies should be reduced by the adoption of rigorous technical and training standards and by second opinion reviews.

Disclosures: Donald Heck—UNRELATED: Consultancy: Stryker; Payment for Lectures (including service on Speakers Bureaus): Stryker. Benjamin Greenberg-UNRELATED: Consultancy: Novartis, Boston Pharmaceuticals, Bayer; Grants/Grants Pending: Chugai, Medimmune, Acorda, Genentech, Patient-Centered Outcomes Research Institute, National Institutes of Health, Guthy Jackson Charitable Foundation*; Payment for Lectures (including service on Speakers Bureaus): Multple Sclerosis Association of America. Carlos Pardo-UNRELATED: Expert Testimony, Comments: expert witness for legal purposes and legal deposition; Grants/Grants Pending: Transverse Myelitis Association, Comments: the Transverse Myelitis Association provides grant support to the Johns Hopkins Transverse Myelitis Center for facilitating patient treatment and research on myelopathies*. Philippe Gailloud-UNRELATED: Consultancy: Codman Neurovascular; Grants/Grants Pending: Siemens Medical*; Stock/Stock Options: Artventive Medical. *Money paid to the institution.

\section{REFERENCES}

1. Riche MC, Reizine D, Melki JP, et al. Classification of spinal cord vascular malformations. Radiat Med 1985;3:17-24 Medline

2. Aminoff MJ, Barnard RO, Logue V. The pathophysiology of spinal vascular malformations. J Neurol Sci 1974;23:255-63 CrossRef Medline

3. Merland JJ, Riche MC, Chiras J. Intraspinal extramedullary arteriovenous fistulae draining into the medullary veins. $J$ Neuroradiol 1980;7:271-320 Medline

4. Hurst RW, Kenyon LC, Lavi E, et al. Spinal dural arteriovenous fistula: the pathology of venous hypertensive myelopathy. Neurology 1995;45:1309-13 CrossRef Medline

5. Jellema K, Canta LR, Tijssen CC, et al. Spinal dural arteriovenous fistulas: clinical features in $\mathbf{8 0}$ patients. J Neurol Neurosurg Psychiatry 2003;74:1438-40 CrossRef Medline

6. Chen J, Gailloud P. Safety of spinal angiography: complication rate analysis in $\mathbf{3 0 2}$ diagnostic angiograms. Neurology 2011;77:1235-40 CrossRef Medline

7. Aminoff MJ, Logue V. Clinical features of spinal vascular malformations. Brain 1974;97:197-210 CrossRef Medline

8. Transverse Myelitis Consortium Working Group. Proposed diagnostic criteria and nosology of acute transverse myelitis. Neurology 2002;59:499-505 CrossRef Medline

9. Merland J, Assouline E, Rüfenacht D, et al. Dural spinal arteriovenous fistulae draining into medullary veins. Clinical and radiological results of treatment (emboliation and surgery) in 56 cases. In: Valk J, ed. Neuroradiology 1985/1986: Proceedings of the XIIIth Congress of the European Society of Neuroradiology, Amsterdam, 11-15 September 1985. Amsterdam: Elsevier; 1986

10. Gailloud P. Diagnostic inefficiency of nonselective spinal angiography (flush aortography) in the evaluation of the normal and pathological spinal vasculature. Curr Probl Diagn Radiol 2016;45:180 -84 CrossRef Medline

11. Pinto A, Brunese L. Spectrum of diagnostic errors in radiology. World J Radiol 2010;2:377-83 CrossRef Medline 